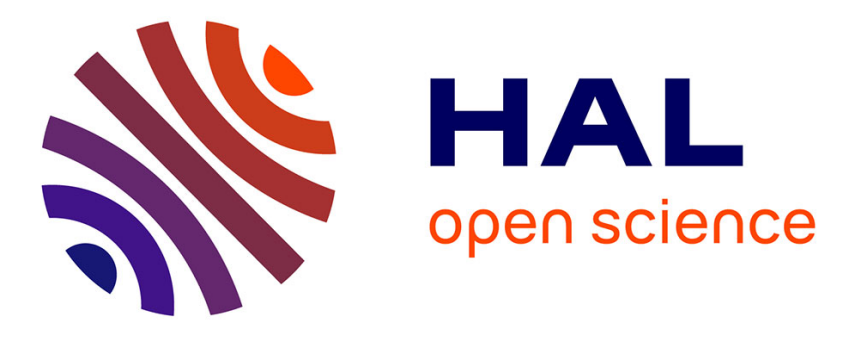

\title{
Optimisation of train speed to limit energy consumption
} Julien Nespoulous, Christian Soize, Christine Funfschilling, Guillaume Perrin

\section{To cite this version:}

Julien Nespoulous, Christian Soize, Christine Funfschilling, Guillaume Perrin. Optimisation of train speed to limit energy consumption. Vehicle System Dynamics, 2022, 60 (10), pp.3540-3557. 10.1080/00423114.2021.1965628 . hal-03322908

\section{HAL Id: hal-03322908 \\ https://hal.science/hal-03322908}

Submitted on 20 Aug 2021

HAL is a multi-disciplinary open access archive for the deposit and dissemination of scientific research documents, whether they are published or not. The documents may come from teaching and research institutions in France or abroad, or from public or private research centers.
L'archive ouverte pluridisciplinaire HAL, est destinée au dépôt et à la diffusion de documents scientifiques de niveau recherche, publiés ou non, émanant des établissements d'enseignement et de recherche français ou étrangers, des laboratoires publics ou privés. 


\title{
Optimization of train speed to limit energy consumption
}

\author{
Julien Nespoulous ${ }^{\mathrm{a}, \mathrm{b}}$, Christian Soize ${ }^{\mathrm{a}}$, Christine Funfschilling ${ }^{\mathrm{b}}$ and Guillaume Perrin ${ }^{\mathrm{c}}$ \\ ${ }^{a}$ Univ Gustave Eiffel, MSME UMR 8208, 5 Bd Descartes, 77454 Marne-La-Vallée, France; \\ b SNCF Innovation \& Recherche, 1-3 Av François Mitterrand, 93574 Saint-Denis, France; \\ c Univ Gustave Eiffel, COSYS, 5 Bd Descartes, 77454 Marne-La-Vallée, France
}

\author{
ARTICLE HISTORY \\ Compiled August 5, 2021
}

\begin{abstract}
The speed profile of a train plays an important role in energy consumption and resulting costs. The industrial objective of this work is thus to develop a method to reduce the energy consumed by a train over a journey by playing on the driver commands (traction and braking forces) while respecting punctuality constraints. A coupling between measured data and simulation is proposed to solve this optimization problem. First, a rigid body approach (Lagrangian formalism) is introduced to characterize the dynamics of each element of the train and their interactions with their environment. In particular, the aerodynamic (including the wind effect), traction, and braking forces are taken into account, and a special attention is paid to the vertical and lateral characteristics of the track as they play a key role on the train dynamics. Secondly, a model for energy consumption and recovery (thanks to dynamic braking) is introduced. Experimental measurements of a high-speed line are then used to estimate the parameters on which the two previous models are based and to validate their predictive capacities. The optimization problem under constraints is finally solved using an evolutionary algorithm where the constraints are implemented using an augmented Lagrangian formalism. The performance of the proposed method in terms of speed optimization and energy consumption reduction is compared to measurements associated with commercial trains.
\end{abstract}

\section{KEYWORDS}

Optimization under constraints, high-speed train, energy saving

\section{Introduction}

The electric energy consumed by a train greatly depends on the driver commands. Indeed, we can measure variations of the energy consumed of around $20 \%$ between two drivers on the same journey. To limit this, some crossing points indicate at which speed and at which time the driver should pass a position. Nevertheless, important variations of the energy consumed are still noticed. Thus, the objective of this work is to propose a strategy to help the driver to find the train controls that will allow him to best meet the punctuality constraints while consuming as little energy as possible. This would particularly be useful when traffic is disrupted.

Several papers have addressed related objectives. Most of them are based on the coupling of two simplified models: one for the train dynamics and one for the energy consumption. In terms of dynamic modeling, rigid body approaches combined with a Lagrangian formalism are mainly considered [1], as well as the projection of the dynamics equations on the longitudinal to the train axis [2]. As for the modeling of the consumed energy, more or less sophisticated 
models can be found in the literature to fit with the reality [3]. The same framework will be used in this work for physical modeling.

These models can then be used to minimize the energy consumed by the train during its journey. The main difficulty lies in the fact that the dynamics and the constraints are nonlinear. To speed up the resolution of the nonlinear dynamic problem, [4 proposes to use the pseudo-spectral method, which projects the continuous equations on orthogonal polynomials. The optimization problem is then solved thanks to a nonlinear programming algorithm. Another approach is to translate the nonlinear dynamic problem and constraints into a mixed integer linear programming problem, which is easier to numerically solve with a linear programming solver [5]. Genetic or dynamic algorithms [6] were also proposed to manage these non-linearities. In this work, we have chosen to use these evolutionary algorithms because of their general and flexible nature.

This type of energy optimization has been used to more precisely study different driving choices: the braking strategy and its impact on energy recovery [7], but also the arbitration between delay-recovery, energy-saving or on-time strategy [8]. Finally, there are works integrating the minimization of rail and wheel wear. Indeed the modifications and the potential multiplication of braking and acceleration phases induced by the minimization of energy could prematurely degrade the system [9].

In this work, we present a method that distinguishes itself from these different works for several reasons. First, the optimization method is applied to the driver commands and not directly on the speed trajectory. Thus, the method can directly be transposed to real high-speed trains. Then, the constraints combine both punctuality (applied on the end speed and position of the train) and physical constraints (applied all along the journey). The model includes both pneumatic and dynamic braking, which is able to recover energy. Those modifications allow a better description of the dynamic behavior of the train. Finally, we use an iterative method to limit the number of evaluations of the cost function, which would otherwise be far too long. A validation process has been carried out by comparing the simulation results with measured data in real traffic conditions.

The implementation of a real-time driver command must be based on the use of a robust model, a fast optimization algorithm, and an appropriate computer. The robust aspects with respect to uncertainties are in progress and are not presented in this paper. The optimization algorithm that is presented is already fast because it is based on the use of a surrogate nonlinear dynamical model (digital twin model) constructed from the full detailed dynamical model of the train. In the optimization algorithm, the computation devoted to each draw of the population of points (last paragraph of Section 3.2 is parallelized. For each draw the surrogate nonlinear dynamic model is used (second paragraph of Section 3.2 and requires, using one core of a CPU, about 30 seconds for the system under consideration (the one described in Section 2.1). Using a GPU instead of a CPU would yield a negligible calculation time with the GPU and consequently, the real-time optimization will be able to be performed without difficulty with an appropriate computer configuration (several GPU's in parallel).

The organization of the paper is the following. Section 2 describes the models that will be used to model the high-speed train longitudinal dynamics and its energy consumption. The optimization problem, its numerical implementation, and the results it can provide, are then presented in Section 3. At last, Section 4 concludes and proposes several prospects for this work. 


\section{High-speed train dynamics and energy consumption}

This section presents the system of interest (a train running at high speed on a dedicated railway track) and proposes models to compute its dynamic behavior and the energy it consumes. The predictive character of these different models is then evaluated by confronting the simulation results with in-line measurements.

\subsection{System description}

In this work, we use a model of a TGV-Dasye that is a commercial high-speed train running on the French Rhin-Rhone high-speed line. This train is composed of eight passengers cars pulled by two engines at the head and tail of the train. Except for the two motor cars, bogies are shared between carriages. The engine bogies are called motor bogies to dissociate them from carrying bogies. Each bogie is composed of two wheelsets. These three bodies (cars, bogies, and wheelsets) are integrated in a rigid body framework to describe their dynamic behavior.

There are three kinds of interactions imposed on the system: the internal, the external, and the driver command interactions.

First, the rigid bodies are linked to each other by suspension joints. These joints can take different forms with linear (springs, dampers) but also nonlinear (bumpstops, bushes, pinlinks elements ...) behaviors. These behaviors are assumed well known and described by their behavior laws. We consider these suspensions as internal to the system because it consists in interactions between rigid bodies.

The external forces regroup all the interactions between the train and its environment. It includes the weight, the aerodynamic forces, and the contact forces. The weight is expressed on each rigid body thanks to the mass and inertia tensor. The aerodynamics can be applied to each moving rigid body, but we neglect it on bogies and wheelsets. In particular, the aerodynamic forces take into account the effect of wind, which has a big influence on the energy consumed by the train [10]. This wind speed is generally decomposed in two terms: the mean speed of wind $\boldsymbol{v}_{w}(t)$, which is forecasted at different positions of the track, and the random fluctuations around this mean value (that can be modeled by a stochastic process). In this work, these fluctuations are neglected and we only keep the mean speed of wind. The mean speed of the wind is then projected on the longitudinal axis defined by the track and can be used to obtain a better approximation of the aerodynamic force. An example of the wind profile that has been used and its projection on the track axis is shown on Figure 1. To model the wheel/rail interaction, the Hertz model is chosen to describe the normal forces and the contact creep forces are approximated thanks to Kalker's wheel/rail theory (see [11, 12] for more details about these contact forces).

Finally, the command forces correspond to traction and braking, as their amplitude is controlled by the driver. Two types of braking are however distinguished, the dynamic and pneumatic braking, because they do not apply to the same subsystems and do not have the same impact on the energy consumed. In the same way as for the traction forces, the dynamic braking is applied to the motor wheelsets and can be simply described as the inversion of the motors. It is therefore particularly interesting as it is able to recover energy and inject it in the catenary. On the contrary, the pneumatic braking only dissipates energy but is applied to each carriage wheelsets.

The driver command $u(t)$, which indirectly controls the train speed, can be modeled by a continuous time-dependent function representing the traction and braking normalized torque. This command is a mathematical modeling of the traction/braking manipulator used by the train drivers. The traction and braking forces capacity are limited by the motor and braking 


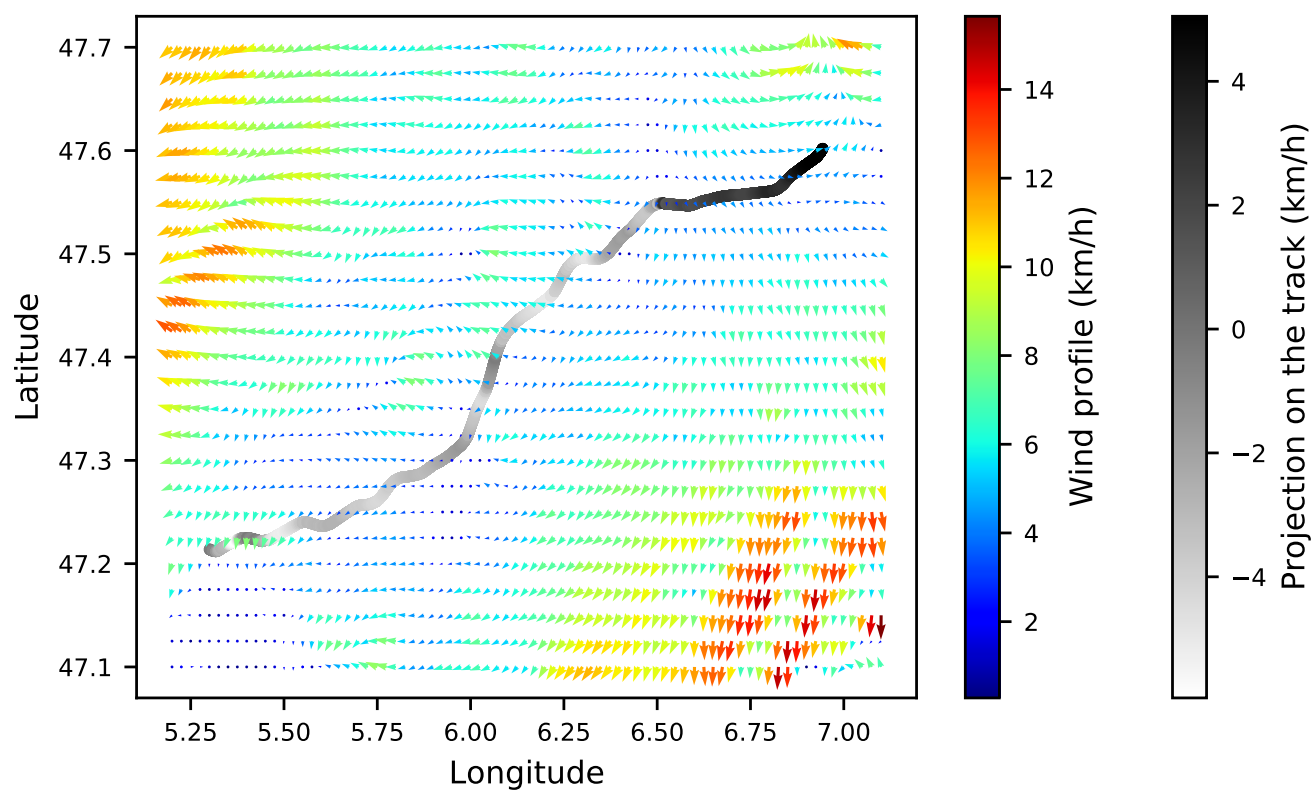

Figure 1. Wind profile on the track.

capacities. In the proposed model, the driver command $u(t)$ takes its values in the interval $[-1,1]$. The bound -1 (resp. 1 ) is the maximum value for the braking (resp. traction) forces, which cannot be exceeded in the model. These maximal traction and braking capacities depend on the train speed. These bounds correspond to longitudinal accelerations that cannot induce passengers comfort and train couplers problems. The jerk of the train is controlled by the time derivative of $u$. In this version of this work, no constraint has been introduced for limiting the jerk. Nevertheless, we have checked that for the optimal driver command found, this derivative stays sufficiently small. If $u(t)$ is positive (respectively negative), the driver uses the motors (respectively brakes). The dynamic braking is prioritized over the pneumatic braking following the braking strategy of in-line trains. Thus, the pneumatic braking is only used for highamplitude braking (for example for emergency stops). For instance, at a time $t$, if $u(t)=-0.8$ the driver is braking using $80 \%$ of the maximal braking capacity. For $u(t)<0$, the traction torque is equal to zero. By defining this control parameter instead of directly working on the train speed, as it is generally done, we get as close as possible to the behavior of the driver who can choose when to use motors or brakes. In addition to being novel, this approach will also facilitate the integration of the obtained results into the on-board computers.

\subsection{Models for the train dynamics and the energy consumption}

\section{Dynamics equations}

A nonlinear $3 D$ dynamic model is constructed from the Lagrangian formalism using a multibody approach applied to each rigid body ( 8 passenger cars, 2 motor cars, 13 bogies, and 26 wheelsets). All the forces that have been listed in Section 2.1 are taken into account in the model (including aerodynamic, contact, and suspension forces) and are adapted to the TGV-Dasye train under consideration. As the objective of this work is to reduce energy consumption under punctuality constraints and speed limitations, the quantities of interest we need to extract from the train dynamics are the evolution of the speed and the 
energy consumption during the simulated journey, but also the arrival time and the final position. An important point is that all these quantities can be derived from the longitudinal dynamics of the train only, which invites us to directly consider the behavior of the whole train. In this work, we have chosen to study the longitudinal dynamics of the whole train whose model is derived from the complete $3 D$ model briefly evoked above. This has been done by summing the Lagrangian of the different rigid bodies and then projecting the Lagrange equations on the longitudinal axis. It should be noted that one of the following step of this work will include the rail and rolling stocks damage in the optimization problem. This objective will impose the use of a complete multibody dynamic model that will be carried out. This is the reason why the complete multibody model is not described in this paper.

To obtain a model whose calculation time is relatively small, several hypotheses are now introduced to simplify the equations. First, it is assumed that the rigid bodies keep the same distance to each other. As a consequence, the internal interactions can be neglected, and the train can be considered as one element evolving at a longitudinal speed denoted by $v(t)$. In particular, the angular speed is assumed to be negligible for the cars and bogies (only the wheelsets turn significantly), which considerably simplifies the expression of the total kinetic energy of the train. In practice, it appears that the rotation term represents approximately $4 \%$ of the longitudinal part. Thus, the kinetic energy $T(v(t))$ is often written, with a corrective term $k^{\text {corr }}=1.04$, as

$$
T(v(t))=\frac{1}{2} m_{t o t} k^{c o r r} v(t)^{2},
$$

with $m_{t o t}$ the total mass of the train. It may vary between two journeys until more or less $5 \%$ of a central value (because of different numbers of passengers in the train). As the total mass will be measured directly on in-line trains in the future, we assume it well known. Thus, we determine it for each series thanks to the experimental measurements.

Secondly, we assume there is no slip between wheels and rail, and that the wheel radius is approximately constant and equal for all the wheels. In this case, the traction and braking torques are equally distributed on each wheelset and can be converted in a global longitudinal force applied to the train. In addition, if we assume that the contact parameters have no influence on the driver maximum command force, the longitudinal components of the different braking at time $t$ can be expressed as functions of the command driver $u(t)$ and the train speed $v(t)$,

$$
\begin{aligned}
& F^{T}(v(t), u(t))=u(t) F_{\max }^{T}(v(t)), \\
& F^{B}(v(t), u(t))=u(t) F_{\max }^{B}(v(t)),
\end{aligned}
$$

where $F_{\max }^{T}$ and $F_{\max }^{B}$ are respectively the maximal traction and braking capacities. Empirical models based on experiments are chosen for these maximal capacities.

Then, the expression of longitudinal running resistance, which regroups both aerodynamic and contact forces is approximated with the Davis formula [13]:

$$
F^{\text {Davis }}\left(v(t), v_{w}(t)\right)=A+B v(t)+C\left(v(t)-v_{w}(t)\right)\left|v(t)-v_{w}(t)\right|,
$$

in which $A, B$ and $C$ are experimental parameters specific to each train. In this expression, coefficient $C$ refers to the aerodynamic air pressure effect, coefficient $B$ mostly comes from the wheel flange friction, and coefficient $A$ refers to the journal friction, the rolling and track 
resistances. In order to improve the running resistance in curve, a correction term is applied to each car $\alpha$ which is in curve under the form:

$$
F_{\alpha}^{\text {Curve }}\left(s_{\alpha}\right)=m_{\alpha} g \frac{k^{\text {Curve }}}{R\left(s_{\alpha}\right)},
$$

with $s_{\alpha}$ the longitudinal abscissa, $m_{\alpha}$ the mass, $g$ the acceleration of gravity, and $R\left(s_{\alpha}\right)$ the curve radius of the track associated with the $\alpha$-th car of the train. As previously, the coefficient $k^{\text {Curve }}$ is determined experimentally and is specific to each train. Finally, the weight of the $\alpha$-th car, projected on the longitudinal axis, is written as

$$
F_{\alpha}^{W}\left(s_{\alpha}\right)=-m_{\alpha} g \sin \left(\theta\left(s_{\alpha}\right)\right),
$$

where $\theta\left(s_{\alpha}\right)$ is the declivity of the track.

With these hypotheses and approximations, the dynamics equation in the longitudinal axis can be written as,

$$
\begin{aligned}
m_{\text {tot }} k^{\text {corr }} \frac{d v}{d t}(t)=F^{T}(v(t), u(t)) & -F^{B}(v(t), u(t))-F^{\text {Davis }}\left(v(t), v_{w}(t)\right) \\
& -\sum_{\alpha} F_{\alpha}^{C u r v e}\left(s_{\alpha}\right)+\sum_{\alpha} F_{\alpha}^{W}\left(s_{\alpha}\right) .
\end{aligned}
$$

Thanks to this equation, which describes the dynamics of the whole train, we can calculate the position and speed of the train all over the journey, and compute the final speed and final position of the train, which will be included as constraints in the optimization problem.

\section{Energy consumption}

From the speed of the train and the driver commands, we can estimate the motor power and the dynamic braking power used by the train. With an appropriate definition of efficiencies, it is then possible to calculate the energy consumed as well as the energy recovered by the system during the braking. Let $\eta_{T}$ and $\eta_{R}$ be the traction and recovery efficiencies. Attention is drawn to the fact that the definition of these two parameters is different. As an example, if we say that the traction efficiency is around $87 \%$, this means that the mechanical power of the motor represents $87 \%$ of the electric power transmitted by the catenary (the rest is lost in the traction chain). On the opposite, the recovery efficiency is the part of mechanical power that is reinjected in the catenary (around $82 \%$ ). The efficiency of the traction chain depends on the efficiency of each of its component. The behavior of these components has not been measured by the constructor for the high-speed train under consideration. Consequently, we have chosen to globally model the efficiency by integrating the whole traction chain. We have observed on other types of trains that the efficiency depends on the train speed and on the longitudinal traction force. As we have not access to the values of the efficiency model for the train used as reference, we have proposed an approximated model that directly depends on the mechanical power, and thus indirectly on the train speed and the traction force. We have verified that the result obtained after the identification is close to the efficiency obtained on other types of train and that the simulated energy consumed was close to the measurements. To take into account this dependence, the following simplified models are proposed for the efficiency coefficients,

$$
\eta_{T}\left(P^{T}\right)=a_{\eta} P^{T}+b_{\eta},
$$




$$
\eta_{R}\left(P^{B}\right)=c_{\eta} P^{B}+d_{\eta}
$$

In these two equations, $P^{T}$ and $P^{B}$ are respectively the mechanical traction and mechanical braking power, whereas $a_{\eta}, b_{\eta}, c_{\eta}$ and $d_{\eta}$ are constants that will also be chosen thanks to experimental measurements.

However, traction and braking are not the only functions that consume energy. Indeed, a part of the energy provided by the catenary is transmitted to auxiliary tools of the train, such as the air conditioner and the cooling system. To take into account this additional consumption, we define the auxiliary power as a constant $P^{a u x}$. Finally, with motor, dynamic braking, and auxiliary power, the energy consumed by the train can be calculated along the journey.

\subsection{Parameter identification}

In addition to potentially affecting the quality of the model, the different assumptions presented in the former sections resulted in the introduction of a series of parameters that need to be carefully adjusted. The first step of the parameter identification consists in performing a sensitivity analysis in order to determine the most important parameters that will be identified, the other ones being fixed to their initial values provided by SNCF, the French railway company, for the TGV Dasye. For this step, eleven parameters have been considered: the resistant force coefficients, the traction and recovery efficiency constants, the auxiliary power, the corrective parameter for the resistant force in curve, and two corrective parameters related to the efficiency of the traction and of the energy recovery. The sensitivity analysis has thus been performed with respect to each one of this eleven parameters fixed to their initial values. The sensitivity of the energy consumed by the train is then performed using a sensitivity interval of $\pm 10 \%$ around their initial values. The results of this analysis show that the mots important parameters are:

- the resistant force coefficients $A, B$, and $C$,

- the traction and recovery efficiency constants $a_{\eta}, b_{\eta}, c_{\eta}$ and $d_{\eta}$,

- the auxiliary power $P^{a u x}$.

From this analysis, we also found that coefficient $C$ and the two coefficients $a_{\eta}$ and $b_{\eta}$ associated with the traction efficiency $\eta_{T}$ played the most important role on the energy consumed during the journey. This is not surprising: the resistant force coefficient $C$ has more impact than $A$ and $B$ because at high speed, the aerodynamic force is preponderant. Moreover, as the traction phases are much more frequent than braking phases, $\eta_{T}$ has a greater effect on the energy consumed than $\eta_{R}$. In the following, these eight influent parameters are called the calibration parameters.

To identify these calibration parameters, measurements were carried out on the French RhinRhône high-speed line (LGV RR), where the train of interest is assumed to run. The characteristics of this line, which are the relative altitude (integration of the declivity $\theta(s)$ ) centered on the starting altitude of the journey, the curvature $R(s)$, and the speed limitation $v^{\max }(s)$ are described in Figure 2. The measurements of the position, the speed, and the energy consumed by the train on different portions of this track are detailed in [3]. Wind forecasts have also been simulated by Meteo France near the track at each hour. As an illustration, a particular wind field and its interpolation along the track are shown in Figure 1. Based on this information, and keeping in mind that the driver commands were unfortunately not recorded during these measurements, the following procedure is now proposed for the estimation of the eight calibration parameters.

- The initial values of the parameters are fixed to the values provided by the train constructor and operator. 

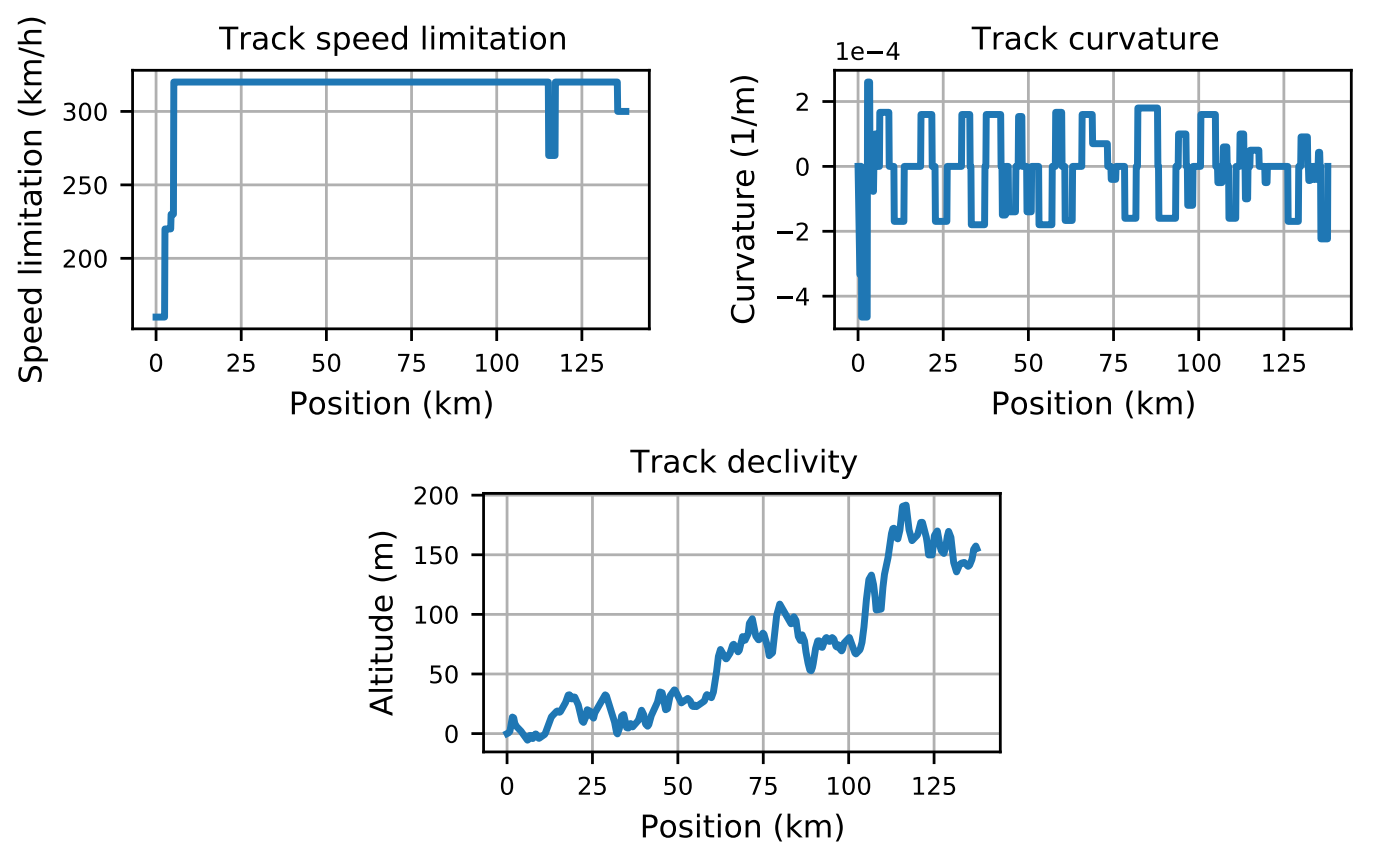

Figure 2. Rhin-Rhône high-speed line description.

- An inverse method is used consisting in calculating the driver commands in such a way that the simulated train trajectory fits with the measured one. In other words, we look for the traction and braking forces so that the simulated and measured speeds and positions are as close as possible.

- Finally, the energy consumed is calculated by simulation and is compared to the measured value.

Comparisons between simulated and measured train speed and energy consumed along the track are shown in Figure 5. We can observe that the inverse method is quite accurate as it is possible to recover driver commands that allow to simulate the same trajectory than the measured one (left plot). Nevertheless, we clearly see on the right plot that working on the driver commands only is not sufficient as the associated simulated energy consumed (dashed line) is not equal to its measured value (dotted line). It should be noted that the energy consumed by the train depends on the traction force and on the speed of the train, which both depend on the driver commands. This energy is estimated by using the nonlinear dynamic equation and the energy consumption model presented in Section 2.2 .

Values for the calibration parameters can however be found such that speed and energy consumed fit relatively well with the measurements. As the driver commands are not unique (they depend on the parameters), the solution of the identification may not be unique as well. To avoid overfitting, the chosen values for the calibration parameters correspond to a compromise between a good representation of the measured speed and energy consumed, and a proximity to the values provided by the constructor. The result of the identification is shown in Figure 3 (solid line). Here, the simulated and measured energy are very close. More precisely, by playing with the values of the calibration parameters, the mean error (calculated as the $L_{2}$-norm between the measured energy consumed along the track and the simulated one) goes from $6.4 \%$ to $3.0 \%$ for 10 different measured journeys.

As an example, the identified values for the resistant force coefficients obtained by using the 

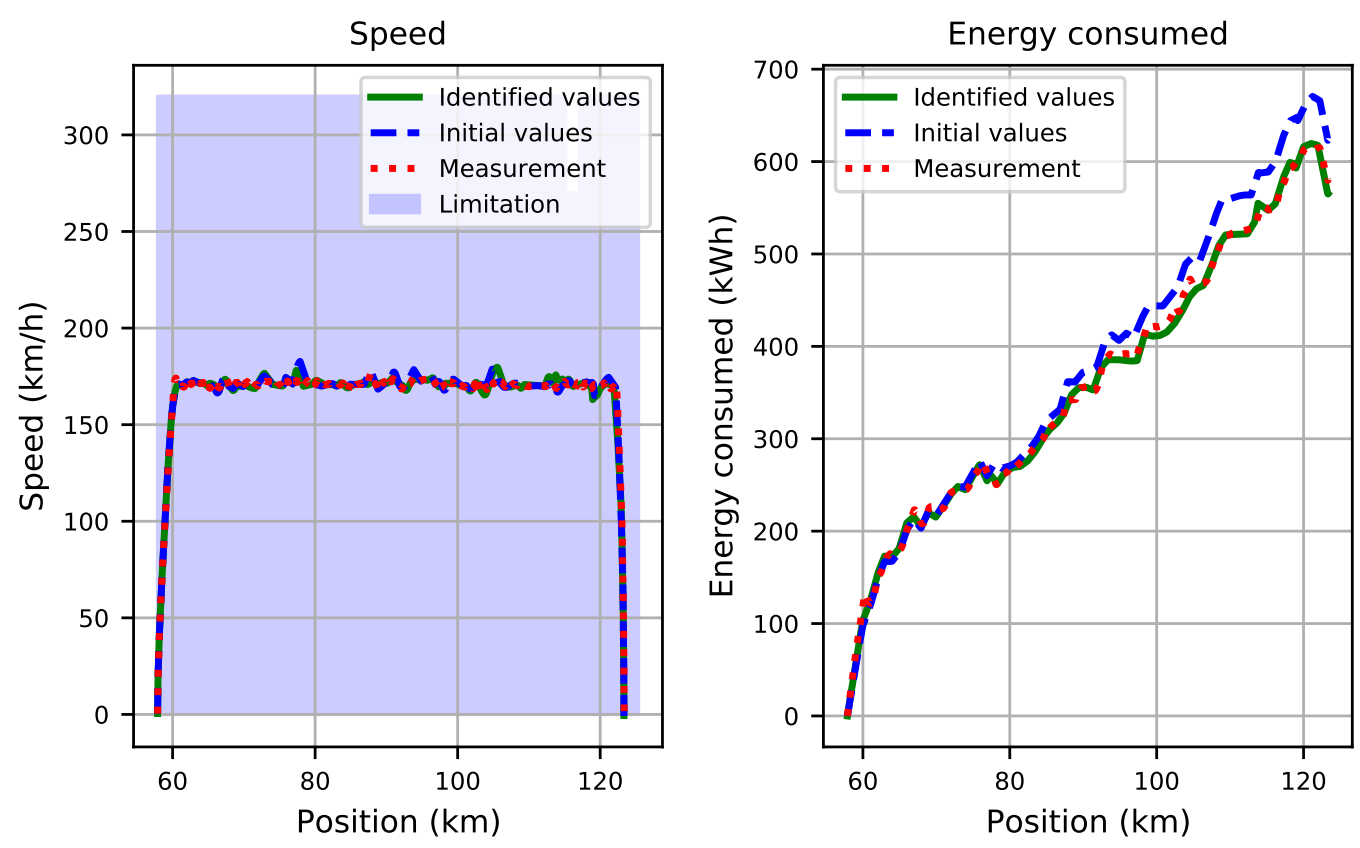

Figure 3. Speed (left figure) and energy consumed (right figure) of the train as a function of its position for the measurements, for the initial and the identified values of the parameters (identification process).

above methodology are $A=2680 \mathrm{~N}, B=31.2 \mathrm{~N} \times s / \mathrm{m}, C=0.427 \mathrm{~N} \times \mathrm{s}^{2} / \mathrm{m}^{2}$. It should be noted that the parameters-identification method that is proposed can be applied to other types of trains.

\subsection{Model validation}

To quantify the relevance of the identified values, comparisons have also been performed for measurements that have not been used during the identification procedure. As an illustration, Figures 4, and 5 compare the energy consumed with initial and identified values for the calibration parameters for data not used during the estimation procedure. Looking at these figures, it can be seen that the predictive capabilities of the model are relatively satisfactory and have been improved compared to the case where the calibration parameters are fixed at their nominal values. More precisely, the mean error goes from $6.9 \%$ to $3.1 \%$ when optimizing these values. For most series ( 8 series out of 10 ), the error is under $3 \%$, which is very encouraging in view of the simplifications introduced, but some measurements have an error slightly over $5 \%$. These differences between simulation and measurements may be due to a shift between speed and energy measurements but can also come from the simplifications listed in the previous section, the presence of specific rolling conditions, such as rain, or uncertainties on the total mass of the train. As an example, running the simulation with a train full of passengers or empty results in variations of the error of around $2 \%$. The auxiliary power, assumed constant, is also a possible source of error. Indeed, it can be dissociated in two parts. The power dedicated to the passenger comfort is approximately constant but the part transmitted to traction or braking auxiliary (cooling system) may vary during the journey. These aspects may be inspected in further studies to improve the quality of the modeling. 

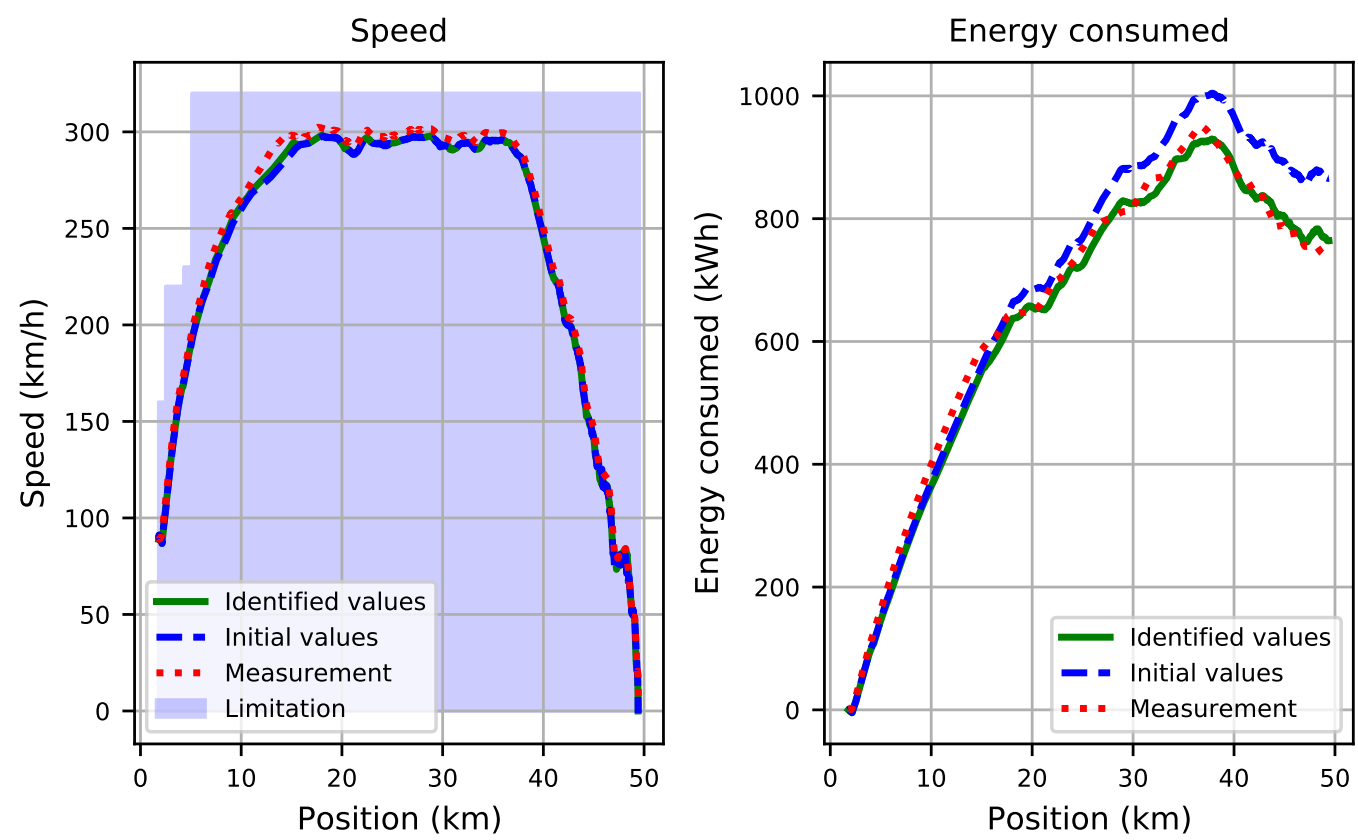

Figure 4. Speed (left figure) and energy consumed (right figure) of the train as a function of its position for the measurements, for the initial and the identified values of the parameters (validation process: measure 1).
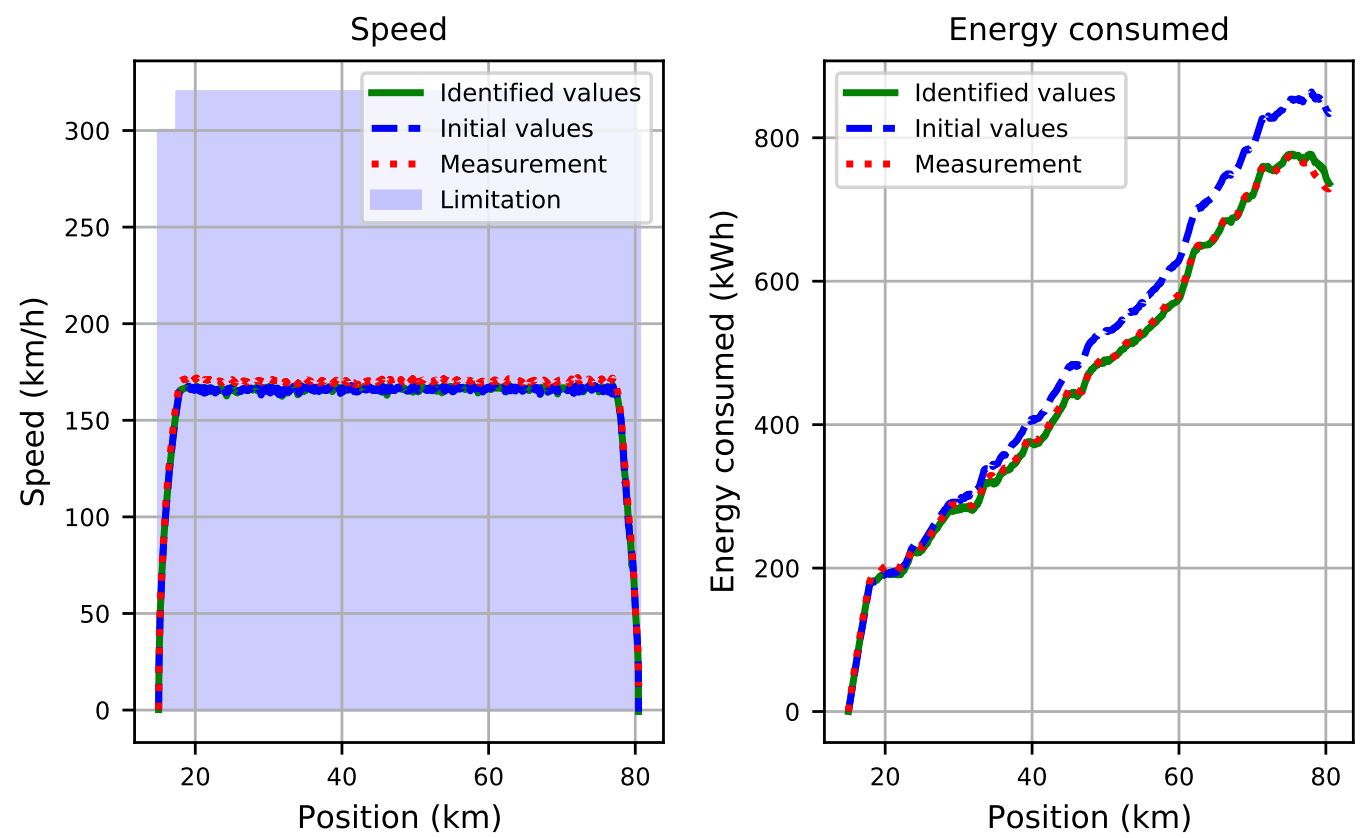

Figure 5. Speed (left figure) and energy consumed (right figure) of the train as a function of its position for the measurements, for the initial and the identified values of the parameters (validation process: measure 2).

\section{Optimization of the driver commands}

Based on the simplified models presented in Section 2, the objective of this section is to present an algorithm to optimize the driver commands in order to minimize the energy consumed by the 
train during a given journey, while verifying punctuality constraints. For this purpose, we will start by presenting the general constrained optimization problem. A number of simplifications will then be listed in order to allow the identification of interesting solutions in a reasonable computation time. The relevance of this simplified problem will finally be illustrated on two real traffic configurations.

\subsection{Definition of the constrained optimization problem}

Definition of the cost function. In this paper, we focus on the impact of the driver command $\{u(t), t \geq 0\}$ on the electric energy consumed by the train during a whole journey. To calculate this energy, we denote by $P^{E}$ the electric power, and we distinguish different configurations depending on the sign of $u$. On the one hand, if $u(t)>0$, the train is in traction phase and the electric power can be written as a function of the traction force $F^{T}$, the traction efficiency $\eta_{T}$, the traction power $P^{T}$, the auxiliary power $P^{a u x}$, and the train speed $v$ :

$$
P^{E}(v(t), u(t))=\frac{1}{\eta_{T}\left(P^{T}\right)} F^{T}(v(t), u(t)) v(t)+P^{a u x} .
$$

On the second hand, if $u(t)<0$, the train is braking, and a part of the dynamic braking power can be recovered:

$$
P^{E}(v(t), u(t))=-\eta_{R}\left(P^{B}\right) F^{D}(v(t), u(t)) v(t)+P^{a u x},
$$

where $\eta_{R}$ is the recovery efficiency and $F^{D}$ is the dynamic braking force. By writing $t_{f}$ the total time of the journey, the total energy consumed by the train is finally given by

$$
f^{E}(v, u)=\int_{0}^{t_{f}} P^{E}(v(t), u(t)) d t .
$$

In some specific cases, it could be interesting to recover energy at special positions on the track or at specific periods (near cities, rush hour for instance). Thus, it might be interesting to express this energy as an integral in space rather than in time, and to weight certain portions of the track for energy recovery. This recovery criterion is however not considered in this study.

Definition of the constraints. So that the driver commands can be used in real configurations, several constraints have to be added to the optimization problem. The first ones concern the comfort of the passengers, and aim to limit acceleration and jerk. As we only consider the longitudinal problem, these constraints are however not restrictive (they could be more limiting when considering lateral and vertical axes). They are therefore not taken into account in the following of the paper. The second group of constraints relates to circulation safety. In our problem, these constraints mainly concern the speed of the train, which can be limited to take into account a high curve radius, a tunnel, a switch or a well identified state of degradation of the track. Let $v^{\max }(s)$ be the speed limitation. The first constraint we will consider is written as

$$
\forall s \quad, \quad v(s) \leq v^{\max }(s),
$$

in which $s$ is the curvilinear abscissa of the track. The last type of considered constraints are the punctuality constraints, which force the train to arrive at the right point $x_{f}$ at the right moment $t_{f}$ with the appropriate speed $v_{f}=0$. In this work, we propose to express these constraints as 
integrals on the train speed $v$ and acceleration $a$ :

$$
\begin{gathered}
\int_{0}^{t_{f}} v(t) d t=x_{f}, \\
\int_{0}^{t_{f}} a(t) d t=v_{f}=0 .
\end{gathered}
$$

Let $\mathcal{C}$ be the space of the driver commands allowing to respect the constraints defined by the equations (12), (13) and (14).

Definition of the constrained optimization problem. If we defined $\{(x(t ; u), v(t ; u)$, $\left.a(t ; u)), 0 \leq t \leq t_{f}\right\}$ as the solution of the dynamic system associated with the driver command $u=\left\{u(t), 0 \leq t \leq t_{f}\right\}$, the optimization problem is written as,

$$
\min _{u \in \mathcal{C}} f^{E}(v(u), u) .
$$

\subsection{Practical solving of the optimization problem}

In order to numerically solve the constrained optimization problem defined by Eq. (15), we need to proceed to several simplifications.

Constant piecewise approximation. Looking for a function $u$ solution of the former optimization problem, means trying to solve an extremely difficult optimization problem because it is defined in a space of infinite dimension. In order to reduce the complexity of the problem, we first propose to limit the search to piecewise constant functions. To this end, we decompose the track into segments of the same length, this length being adapted to the available calculation capacity. The greater the number of segments, the better it is possible to adapt the train speed to the track design and constraints, but the higher the numerical cost. For instance, this length could be chosen around $\delta_{s}=200 \mathrm{~m}$ for journey of about one hundred kilometers.

Solving the dynamic equations. To solve the nonlinear system defined by Eq. (6), we use the Runge Kutta scheme at the order 4 . The associated time step $\delta_{t}$ is chosen equal to $0.5 \mathrm{~s}$ according to a convergence analysis. For this value of $\delta_{t}$, this scheme gives a good precision of the results in an acceptable calculation time.

Perturbation method. As the punctuality constraints can only be evaluated at the end of the dynamic calculation, most of the tested trajectories will not respect these constraints. In order to avoid a too high rejection rate, and thus very high computation costs, we propose in this paper to test only new driver commands under the form of a deviation around a trajectory verifying the punctuality constraints. In other word, if $\underline{u}$ is a driver command allowing to respect the punctuality constraints, it is assumed that there is a good chance that, for $\Delta u$ small with respect to $\underline{u}$, the command $u=\underline{u}+\Delta u$ will be close to respect the constraints. In that case, if $v=\underline{v}+\Delta v$ and $a=\underline{a}+\Delta a$ are the train speed and train acceleration associated with $u$, whereas $\underline{v}$ and $\underline{a}$ are the train speed and train acceleration associated with $\underline{u}$, we have

$$
\int_{0}^{t_{f}} \underline{v}(t) d t=x_{f} \quad, \quad \int_{0}^{t_{f}} \underline{a}(t) d t=0,
$$


such that the new punctuality constraints are rewritten as,

$$
\int_{0}^{t_{f}} \Delta v(t) d t=0 \quad, \quad \int_{0}^{t_{f}} \Delta a(t) d t=0 .
$$

In practice, if we randomly choose $\Delta u$, even if the constraints have a chance to be almost verified, the chances are very low if not zero that they are completely verified. To be able to better compare two driver commands, we propose a double adaptation of the train trajectory. If the train stops before the arrival point, we stop the calculation to avoid negative speed and the constraint on the final position is not verified. On the opposite, if the train is assumed to stop after the arrival point, we stop the calculation at time $t_{f}$. In this situation, both constraints on final speed and final position are not respected.

Optimization algorithm. In order not to limit the research to the immediate surroundings of the initial solution without drawing a huge number of driver command perturbations, we need to use the information given by the dynamic responses to guide the choice of the perturbations. This is how the CMA-ES method works, which we choose to solve the optimization problem under constraints. For this algorithm, to start the calculation, we need to define a starting point, a starting amplitude, and several hyperparameters (see [14 for further details about this method), the constraints being implemented as a penalization of the cost function with Lagrangian multipliers. Among these hyperparameters, we can mention the size of the population, the number of draws selected, and the adaptation coefficient. Then, at each iteration, the method follows several steps, which can be summarized as follows.

- Draw a population of points following the direction given by the covariance matrix around a starting point.

- Estimate the cost function and the constraints for each generated point.

- Select the best points.

- Update the Lagrangian coefficients associated with the constraints.

- Update the covariance matrix and the starting point in order to find a better direction for the next draws.

For the considered application, this method has allowed us to find very interesting solutions with a relatively low number of iterations.

\subsection{Presentation of the optimization results}

The optimization procedure we have proposed in the former section is run for the optimization of the driver command under punctuality and safety constraints. The values of the cost function and constraints are kept as the algorithm is iterated. The simulation is run over the LGV Rhin-Rhône track to verify the quality of the optimal trajectory compared with the energy measurements. Figure 6 displays the cost and constraints distributions represented with box plots of the population with respect to the number of iterations. The final position and speed constraints are expressed relatively to the expected ones. This figure gives a visual interpretation of the random search strategy. In fact, during the first iterations, we see that the standard deviation is important. This means that the method searches roughly a direction to explore. After few iterations, the energy consumed by the train begins to decrease: the method has found an interesting energy-saving direction. However, the punctuality and speed limitation constraints are not respected yet. Then, around 50 iterations, the Lagrangian penalization takes more importance and a new direction is defined. This has an impact on the energy consumed that increases slightly but also on the constraints that are closer to be respected. This method continues until the standard deviation reaches the threshold defined 

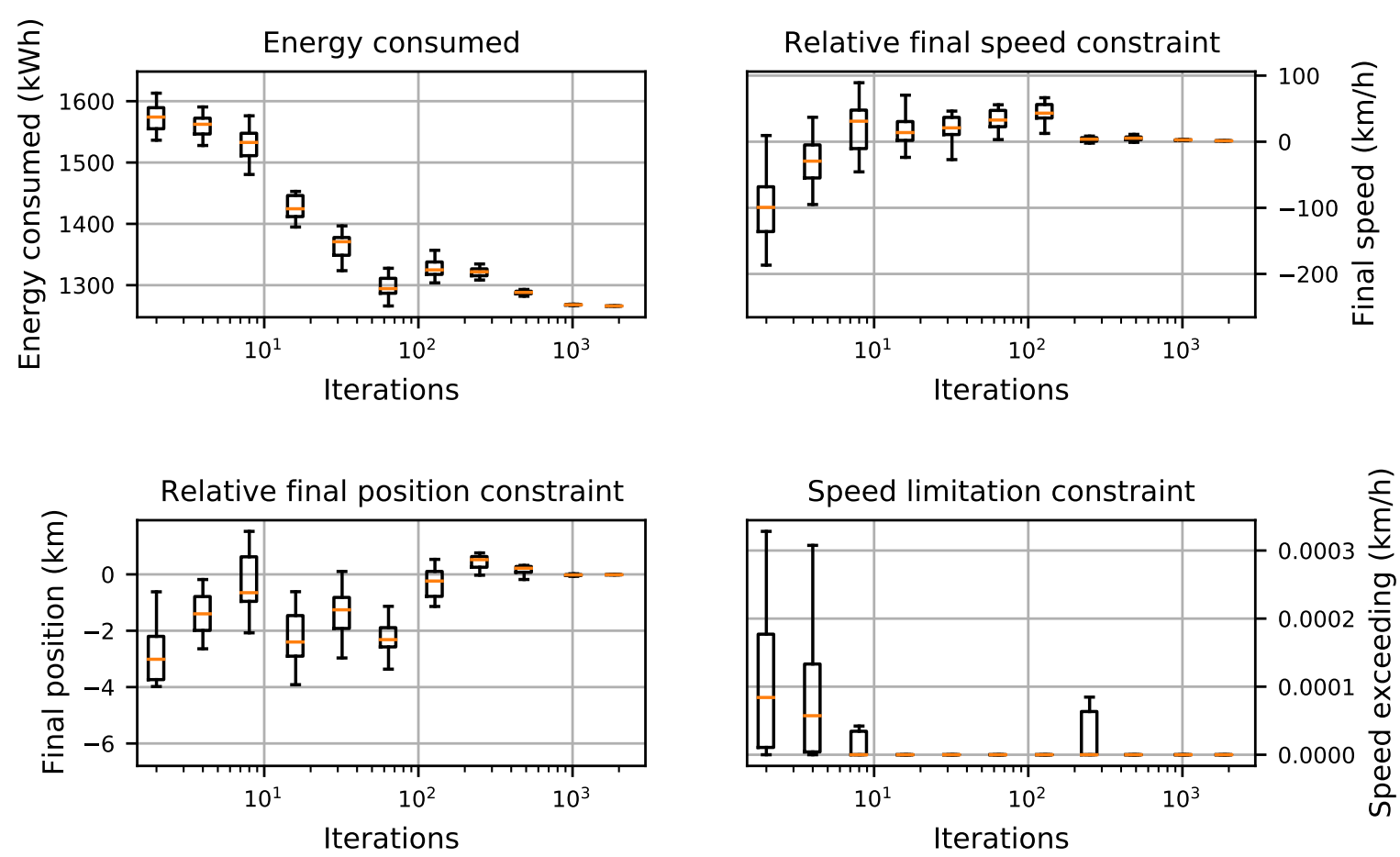

Figure 6. Random draws cost and constraints (LGV Rhin-Rhone).

as a hyperparameter. We choose it in order that the solution obtained at the end of the optimization calculation is converged.

The quality of the optimal solution is then verified by comparing it with measurements. Thus, left Figure 7 shows a comparison of the speed between the measured and optimal trajectories. It can be observed that the optimal trajectory reduces the maximum speed of the train when it is possible but brakes later to stay on time. Indeed, reducing the maximal speed of the train leads to a reduction of the resistant force, thus a reduction of the energy consumption, but the punctuality constraint imposes that the measured and simulated trajectories arrive on the same time. In order to reduce the energy consumption but still to arrive on time, the optimal solution is to decrease the maximal speed of the train and brake later. This is not clearly shown in Figure 7 but the measured speed of the train is around $25 \mathrm{~km} / \mathrm{h}$ during almost $1 \mathrm{~km}$ at the end of the journey whereas the optimal solution brakes at the last moment, this is why both trajectories respect the punctuality constraint. If the punctuality constraint has been tighter, braking later would not have been sufficient and the solution would have been to increase slightly the maximum speed. Right Figure 7 displays the associated energy consumed. It can be seen that the solution is effectively better and consumes around $20.9 \%$ less energy. Thus, the method is interesting to reduce the energy consumed by trains. It can also be observed that, during the first part of the journey, the energy consumed soars, which corresponds to the acceleration parts. Then, during the second part, the energy consumed still increases but slightly, which coincides with the constant speed part of the journey. The energy consumed decreases, which means that the train is braking and recovering energy. The trajectory and energy consumed may be more precise by choosing a lower discretization path $\delta_{s}$, which should improve the energy saving. We can observe this spatial discretization path on the optimal result because the solution is not smooth and seems to be defined piecewise. 

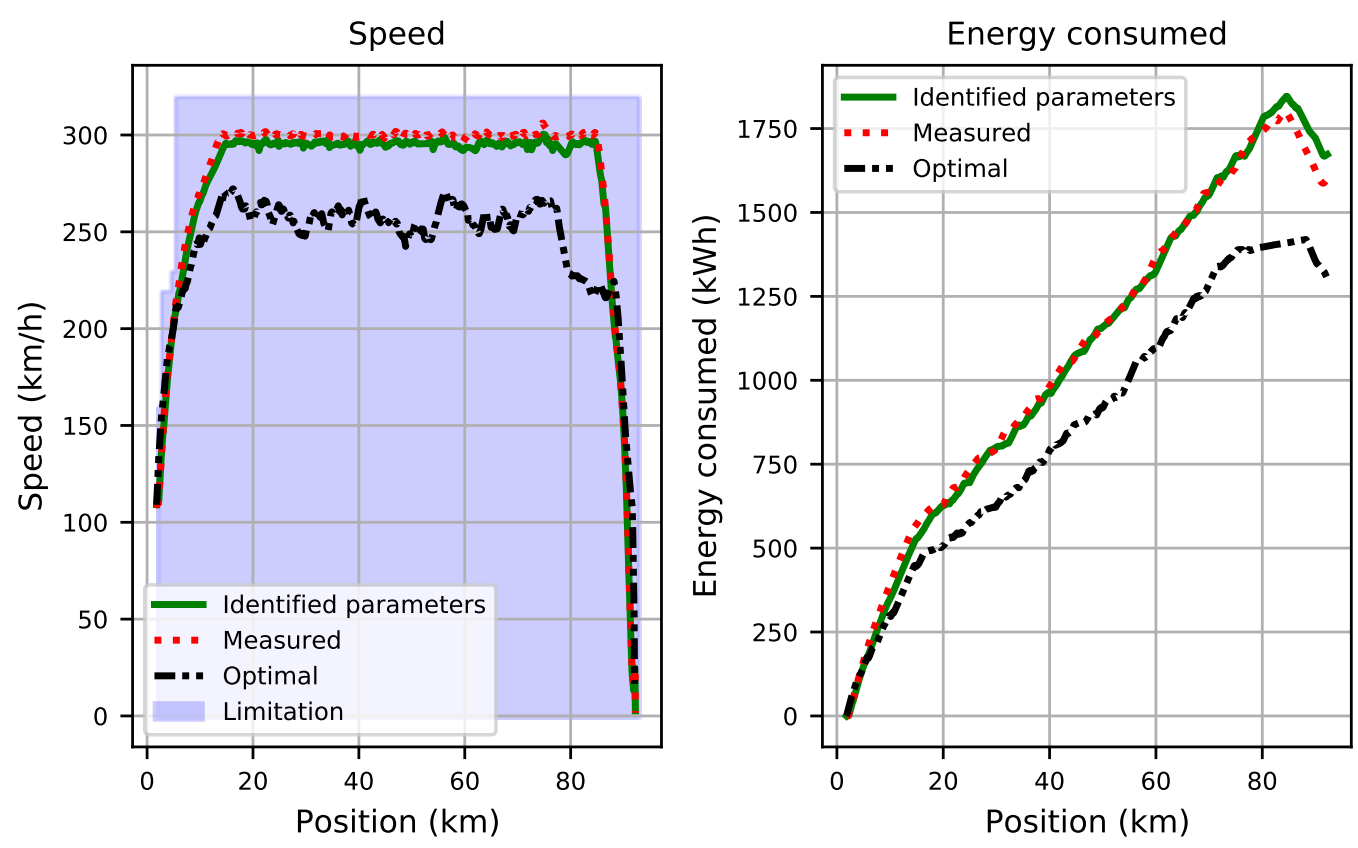

Figure 7. Optimized solution (LGV Rhin-Rhone).

Finally, if we compare the trajectory with the declivity of the track, it can be understood why the speed is not constant once the train reaches its maximal speed. Indeed, in term of energy, it is not efficient to accelerate during a slope if we need to brake when going down. Thus, before the kilometer 60 , it can be seen that the train accelerates and takes advantage of its inertia to climb the slope without accelerating more. This is shown in Figure 8 that displays the optimal trajectory of the optimization run on another journey (LGV est) in the left figure and the associated altitude of the track in the right figure. Here, the arrival time is set arbitrarily so that the train needs to reach its maximal speed for most of the journey to be on time. Thus, we can highlight the efficiency of the maximal speed constraint, which is well respected. Despite this, we still observe that before each positive declivity (kilometers 31, 37 or 68), the optimal trajectory have important speed, which confirms the previous observations. The punctuality and the speed limitation constraints are respected as we can see on the trajectory (left plot in Figure 7). In this work, the comfort and security constraints have not been taken into account but the Lagrangian penalization offers the capacity to add as many constraints as we need. The only impact will be on the time calculation of the method, which will need more time to converge to an optimal trajectory.

\section{Conclusion}

In this paper, a model has been proposed to describe the high-speed train dynamics among the longitudinal axis. The parameters have been identified and the model has been validated thanks to measurements carried out on a real high-speed line. Then, the optimization problem has been defined and numerically solved. Finally, the obtained results have been analyzed and compared it to in-line train.

The method allows the energy consumed to be reduced. It could be used to define an optimal trajectory on each journey to help drivers following the most energy saving trajectory. This application of the method takes even more importance when it is known that the automatic 

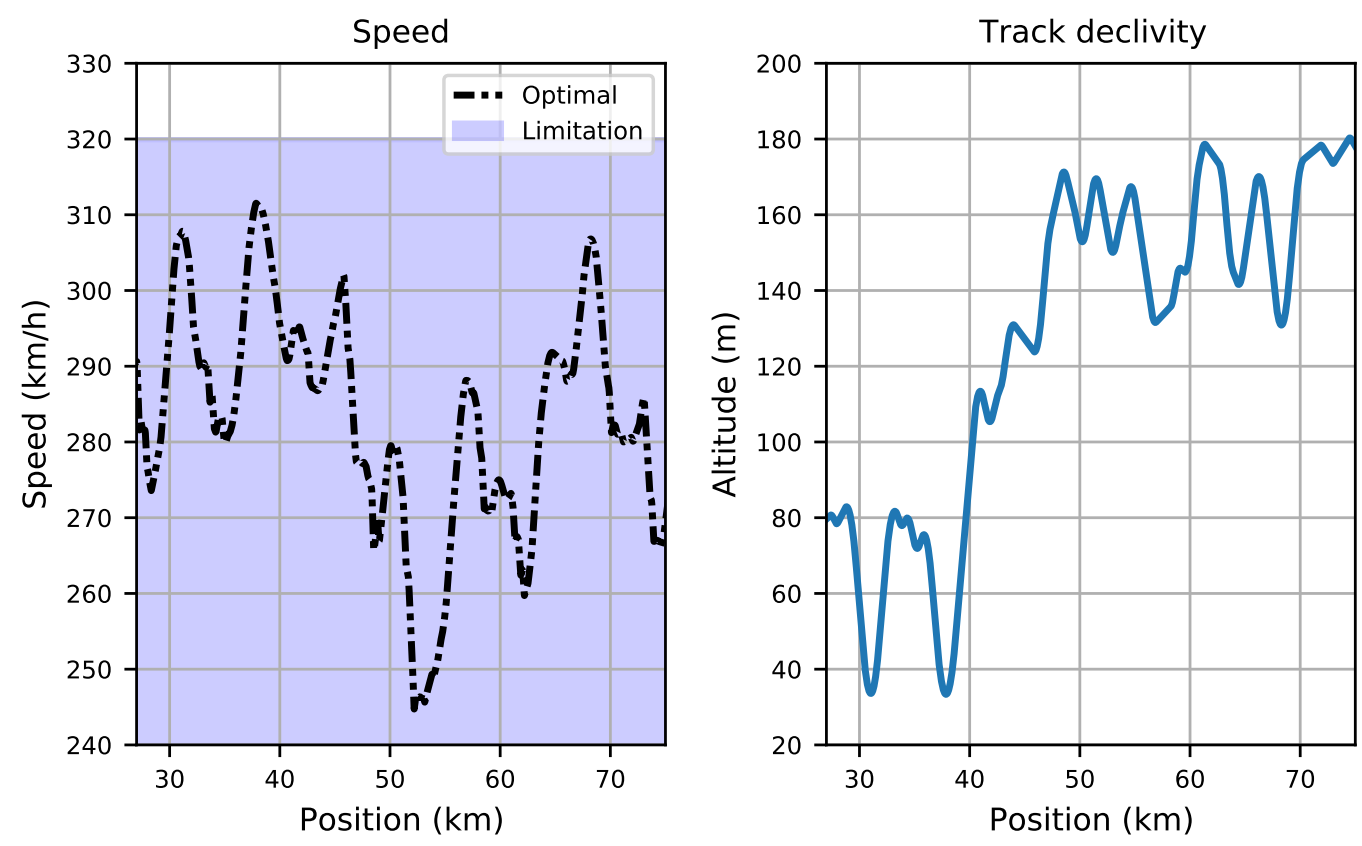

Figure 8. Optimal solution and associated altitude of the track (LGV est).

train is a technology being developed today. It will need a proper trajectory definition as the driver will probably no longer be in the train.

To complete the method, we may introduce other objectives. The optimal trajectory obtained as a result does not take into account other important stakes of railway world. As an example, the wear of wheels and rolling stokes might be fasten if all trains follow the optimal trajectory. This multi-objective aspect will be inspected in further works. The uncertainties of the model parameters is also another further development as we saw that they impact the model quality. Taking it into account allows a better modeling of the physical system and thus improves the optimal trajectory we may find.

\section{References}

[1] Bosso N and Zampieri N. Long train simulation using a multibody code. Vehicle System Dynamics. 2017; 55:552-570.

[2] Wu Q, Spiryagin M and Cole C. Longitudinal train dynamics: an overview. Vehicle System Dynamics. 2016; 54:1688-1714.

[3] Bosquet R. Modélisation énergétique et identification des trains pour l'écoconception des lignes ferroviaires à grande vitesse. Doctoral Thesis (in French), University of Nantes, 2015, https://tel.archives-ouvertes.fr/tel-01201921/document.

[4] Wang P and Goverde RMP. Multiple-phase train trajectory optimization with signaling and operational constraints. Transportation Research Part C: Emerging Technologies. 2016; 69:255-275.

[5] Wang Y, De Schutter B, Ning B, Groot N and van den Boom TJJ. Optimal trajectory planning for trains using mixed integer linear programming. 14th International IEEE Conference on Intelligent Transportation Systems (ITSC). 2011; 1598-1604.

[6] Scheepmaker GM, Goverde RMP and Kroon LG. Review of energy-efficient train control and timetabling. European Journal of Operational Research. 2017; 257:355-376. 
[7] De Martinis V and Gallo M. Models and Methods to Optimise Train Speed Profiles with and without Energy Recovery Systems: A Suburban Test Case. Procedia - Social and Behavioral Sciences. 2013; 87:222-233.

[8] Wang P and Goverde RMP. Multi-train trajectory optimization for energy efficiency and delay recovery on single-track railway lines. Transportation Research Part B Methodological. 2017; 105:340-361.

[9] Conti R, Galardi E, Meli E, Nocciolini D, Pugi L and Rindi A. Energy and wear optimisation of train longitudinal dynamics and of traction and braking systems. Vehicle System Dynamics. 2015; 53:651-671.

[10] Quost X. Modélisation de l'effet du vent sur les trains à grande vitesse, Doctoral Thesis (in French), Ecole Centrale de Lyon, 2005, https://bibli.ec-lyon.fr/exl-doc/xquost.pdf

[11] Liu B and Bruni S. Comparison of wheel-rail contact models in the context of multibody system simulation: Hertzian versus non-Hertzian. Vehicle System Dynamics. 2020; 0:1-21.

[12] Spiryagin M, Polach O and Cole C. Creep force modelling for rail traction vehicles based on the Fastsim algorithm. Vehicle System Dynamics. 2013; 51:1765-1783.

[13] Davis WJ. The tractive resistance of electric locomotives and cars. Editor SChenectady N.Y., General Electic, 1926 ( Reprint of General Electric Review, v.xxix, no. 10, pages 685-707, October, 1926).

[14] Hansen N. The CMA evolution strategy: a tutorial. 2016. ArXiv e-prints, arXiv:1604.00772v1 [cs.LG], 4 April 2016 and https://hal.inria.fr/hal-01297037. 